\title{
SECULAR EVOLUTION OF THE SOMBRERO GALAXY.
}

\author{
ERIC EMSELLEM
}

Sterrewacht Leiden

Postbus 95132300 Leiden, The Netherlands
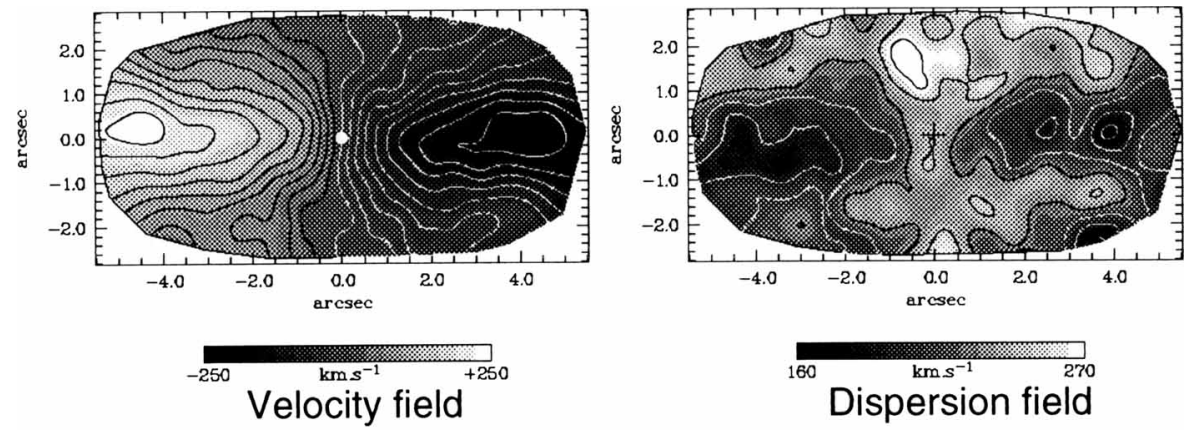

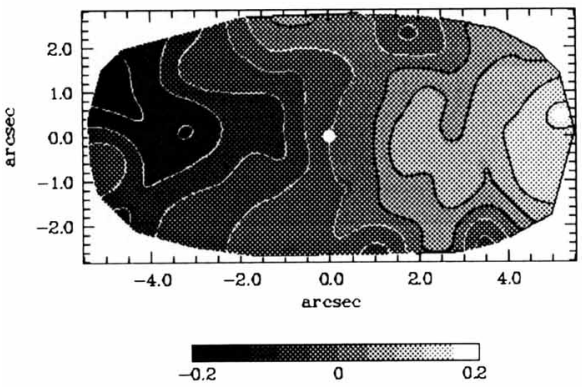

h3

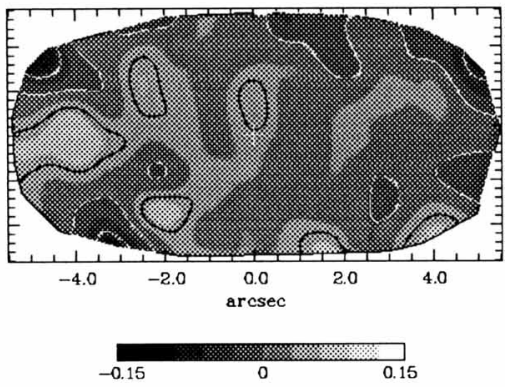

h4

Figure 1. TIGER maps of the stellar velocities, velocity dispersions and the Gauss-Hermite coefficients $\left(h_{3}, h_{4}\right)$ of the central region of M 104.

We present several pieces of evidence that there was an interaction between a bar, which is now dissolved (presumably by a small merger event Emsellem 1995, A\&A in press), and the interstellar medium (gas/dust) in the Sombrero Galaxy (M 104). This study has been achieved by combining new photometric and 2D spectroscopic data (HRCAM, TIGER - Fig. 1) with realistic models (a 3D spatial model of the dust distribution taking into account light scattering, and self consistent two-integral models). Such secular evolution of galaxies driven by bars may be a very common process (Friedli \& Benz 1993, A\&A 268, 65). 